\title{
Daily fraction dose recalculation based on rigid registration using Cone Beam CT
}

\author{
Courtney Bosse ${ }^{1,2}$, Rachel Tuohy1, 2, Panayiotis Mavroidis ${ }^{1,2}$, Zheng Shi ${ }^{1,2}$, Richard Crownover ${ }^{1,2}$, \\ Alonso Gutierrez ${ }^{1,2}$, Niko Papanikolaou ${ }^{1,2}$, Sotiri Stathakis ${ }^{1,2}$ \\ ${ }^{I}$ Department of Radiation Oncology, University of Texas Health Science Center at San Antonio, San Antonio, TX, USA. \\ ${ }^{2}$ Cancer Therapy and Research Center, San Antonio, TX, USA.
}

Received March 19, 2014; Published Online April 08, 2014

[Presented at the Young Investigator's Symposium at the 2014 Annual Meeting of Southwest Chapter of American Association of Physicists in Medicine (AAPM) in San Antonio, Texas, USA]

\section{Conference Proceeding}

\begin{abstract}
Purpose: To calculate the daily fraction dose for CBCT recalculations based on rigid registration and compare it to the planned CT doses.

Methods: For this study, 30 patients that were previously treated (10 SBRT lung, 10 prostate and 10 abdomen) were considered. The daily CBCT images were imported into the Pinnacle treatment planning system from Mosaic. Pinnacle was used to re-contour the regions of interest (ROI) for the specific CBCT by copying the contours from the original CT plan, planned by the prescribing physician, onto each daily CBCT and then manually reshaping contours to match the ROIs. A new plan is then created with the re-contoured CBCT as primary image in order to calculate the daily dose delivered to each ROI. The DVH values are then exported into Excel and overlaid onto the original CT DVH to produce a graph.
\end{abstract}

Results: For the SBRT lung patients, we found that there were small daily volume changes in the lungs, trachea and esophagus. For almost all regions of interest we found that the dose received each day was less than the predicted dose of the planned CT while the PTV dose was relatively the same each day. The results for the prostate patients were similar, showing slight differences in the DVH values for different days in the rectum and bladder but similar PTV.

Conclusion: By comparing daily fraction dose between the re-contoured CBCT images and the original planned CT show that PTV coverage for both prostate and SBRT, it has been shown that for PTV coverage, a planned CT is adequate. However, there are differences between the dose for the organs surrounding the PTV. The dose difference is less than the planned in most instances.

Presenting author: Courtney Bosse; Department of Radiation Oncology, University of Texas Health Science Center at San Antonio, San Antonio, TX, USA.

Cite this article as:

Bosse C, Tuohy R, Mavroidis P, Shi Z, Crownover R, Gutierrez A, Papanikolaou N, Stathakis S. Daily fraction dose recalculation based on rigid registration using Cone Beam CT. Int J Cancer Ther Oncol 2014; 2(2):020217. DOI: 10.14319/ijcto.0202.17 


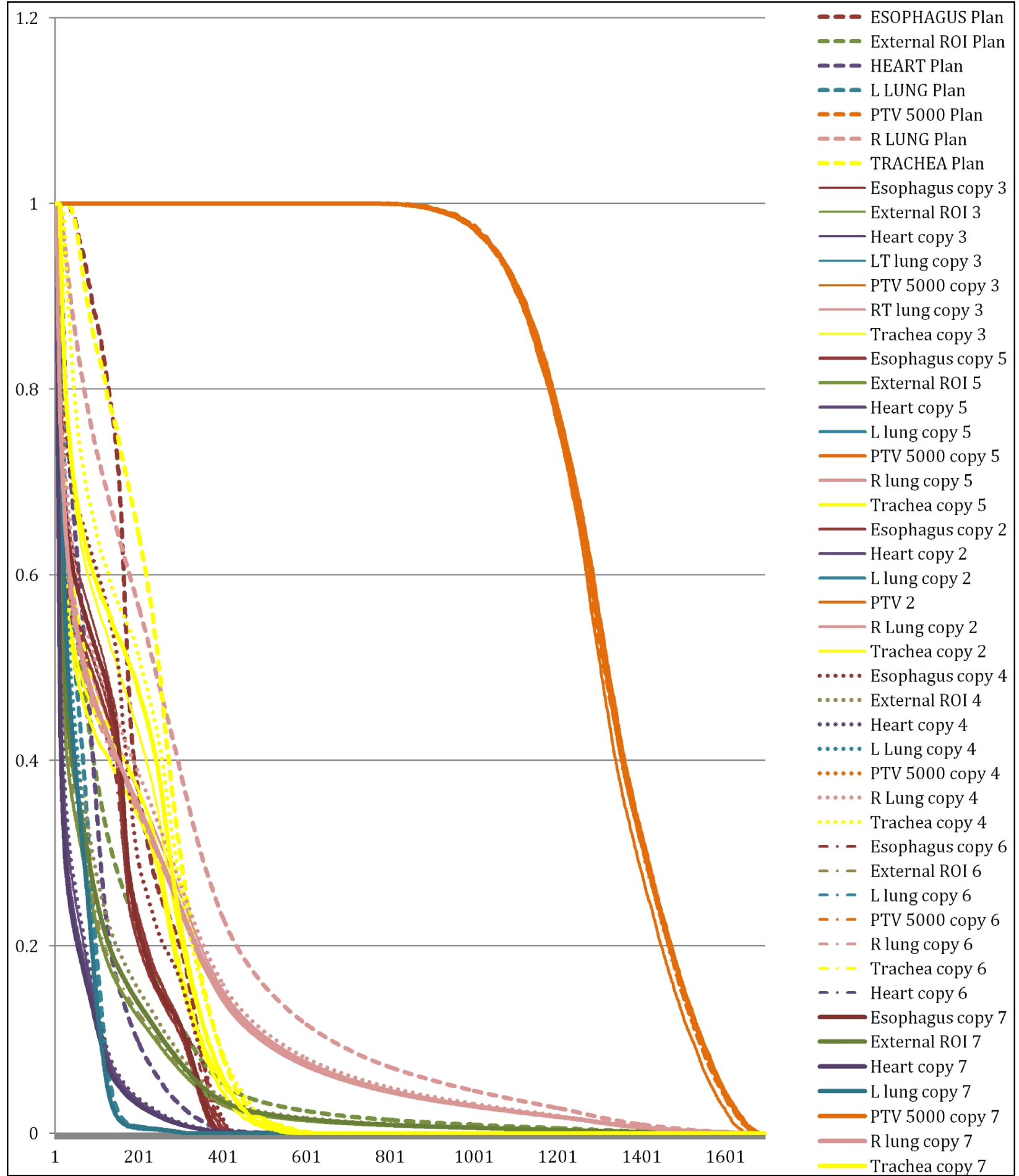

FIG. 1: Daily fraction dose recalculation based on rigid registration using Cone Beam CT. 